\title{
Direct and indirect co-culture of bone marrow stem cells and adipose-derived stem cells with chondrocytes in 3D scaffold-free culture
}

Loraine L.Y. Chiu ${ }^{1,2+}$, Juares Bianco ${ }^{3,4 \dagger}$, Renata Giardini-Rosa ${ }^{3,4}$, Kristina Collavino ${ }^{1,2}$ and Stephen D. Waldman ${ }^{1,2^{*}}$

*Correspondence: swaldman@ryerson.ca

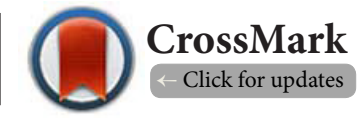

'These authors contributed equally to this work.

'Department of Chemical Engineering, Ryerson University, Canada.

${ }^{2}$ Li KaShing Knowledge Institute, St. Michael's Hospital, Canada.

${ }^{3}$ Human Mobility Research Centre, Kingston General Hospital and Queen's University, Kingston, Canada.

${ }^{4}$ Department of Molecular Biology, Hematology and Hemotherapy Center (Hemocentro), University of Campinas (UNICAMP), Campinas, Brazil.

\begin{abstract}
Background: The use of chondrocytes for cartilage tissue engineering is hampered by the limited number of chondrocytes that can be harvested and potential dedifferentiation during cell expansion. While stem cells is a promising approach to create a large population of differentiated cells, multiple growth factors are typically required for differentiation. Alternatively, co-culturing of stem cells with mature chondrocytes can induce differentiation. However, it is not clear which stem cell population and co-culturing method best supports chondrogenesis. While co-culture of stem cells with chondrocytes has been extensively shown to improve chondrogenesis in general, results from previous reports were convoluted by the use of $2 \mathrm{D}$ culture or scaffold materials, resulting in discrepancies with the comparison between direct and indirect cocultures.
\end{abstract}

Methods: The purpose of this study was to investigate the extent of chondrogenic differentiation of direct and indirect co-culture of bone marrow stem cells (BMSCs) or adipose-derived stem cells (ASCs) with mature chondrocytes in 3D scaffold-free culture. For direct co-culture, cell pellets were created by centrifugation, consisting of chondrocytes alone or different proportions of stem cells to chondrocytes. For indirect co-culture, cell pellets of chondrocytes or stem cells were created individually and cultured with a separation by a trans-well membrane. Chondrogenic differentiation potential was assessed by quantification of DNA, GAG and collagen contents, as well as collagen I, collagen II and Safranin-O staining. Statistical significance was analyzed using one-way ANOVA with Tukey's post-hoc tests.

Results: Direct co-culture of chondrocytes with BMSCs resulted in superior chondrogenesis compared to all other co-culture methods. Cultures with the ratio of 3:1 BMSCs to chondrocytes stained positive for chondrogenic markers and displayed a uniform deposition of cartilaginous extracellular matrix. In addition, the extent of matrix deposition in direct BMSC co-cultures were comparable to growth factor differentiated BMSCs.

Conclusions: Thus, BMSCs appear to be superior to ASCs in their differentiation capacity during coculture and a direct co-culture with chondrocytes (3:1 ratio) may be a feasible strategy for cartilage tissue engineering.

Keywords: Cartilage tissue engineering, co-culture, bone marrow stem cells, adipose-derived stem cells, chondrogenic differentiation, scaffold-free

\section{Introduction}

Damage to joint cartilage is a challenge in orthopedic medicine because of the limited self-repair potential of this tissue [1]. While cell-based therapies, such as Autologous Chondrocyte

(C) 2016 Waldman et al; licensee Herbert Publications Ltd. This is an Open Access article distributed under the terms of Creative Commons Attribution License (http://creativecommons.org/licenses/by/3.0). This permits unrestricted use, distribution, and reproduction in any medium, provided the original work is properly cited. 
Implantation (ACl), has shown some success for repairing cartilage defects [1], recent efforts have focused on the development of tissue engineered cartilage. Although several different strategies have been proposed to engineer cartilaginous tissue, these approaches rely on a large population of chondrocytes to develop sufficient quantities of tissue in vitro [2-5]. However, as only a limited number of chondrocytes can be isolated from a cartilage biopsy and these cells tend to dedifferentiate under routine cell expansion $[1,6]$, there is a need for other cell sources in cartilage tissue engineering.

The use of stem cells is advantageous due to their demonstrated proliferative and regenerative capacities. However, these cells typically require multiple growth factors such as transforming growth factors beta 1 and 3 (TGF- $\beta 1$, TGF- $\beta 3$ ) and bone morphogenetic protein 6 (BMP-6) to support chondrogenic differentiation [7-10]. An alternative approach has been to co-culture stem cells with mature cells to drive differentiation towards specific lineages in the absence of growth factors [11]. In cartilage tissue engineering, previous studies have investigated the effect of co-culturing of bone marrow mesenchymal stem cells (BMSCs) or adipose derived stem cells (ASCs) with mature chondrocytes with varying results. For example, several studies have demonstrated chondrogenic differentiation of BMSCs in direct co-culture with mature chondrocytes both in vitro $[6,12]$ and in vivo [13]. However, differential effects have been reported during the comparison of direct versus indirect co-culturing of BMSCs and mature chondrocytes. Zuo et al., [14] found improved chondrogenesis in direct coculture whereas Levorson et al., [15] reported that indirect co-culture was superior in terms of chondrogenic extracellular matrix accumulation. Similarly, although both direct [16] and indirect [17] co-culturing of ASCs with mature chondrocytes have been shown to induce chondrogenesis, Lai et al., [18] reported improved chondrogenesis with direct co-culture compared to indirect co-culture. While these discrepancies may be attributable to the differences in the species from which the cells were isolated, the proportions of stem cells to mature chondrocytes in the cultures and/or the culturing method (2D culture or 3D biomaterial scaffolds), no direct comparisons between the co-culturing of BMSCs and ASCs have been reported. Thus, the purpose of the present study was to compare the direct and indirect co-cultures of BMSCs and ASCs with chondrocytes as well as to determine a suitable ratio of stem cells to chondrocytes in 3D scaffold-free culture.

\section{Methods}

\section{Cell isolation}

Bone marrow, cartilage and fat were extracted from nine adult cow legs obtained from a commercial slaughterhouse (Brian Quinn's Meats, Kingston, ON). Cells from the same donors were used in co-culture experiments.

Isolation of bovine bone marrow mesenchymal stem cells The fascia and muscle were removed from the cow leg, expos- ing the bone. The bone was then cut on the mid-diaphysis area using a sterile bone saw, and the marrow was removed using a spatula. Equal volumes of bone marrow was mixed with $0.5 \%$ collagenase VIII in Krebs-Ringer buffer containing $1 \mathrm{mM}$ glucose, $5 \mathrm{mM} \mathrm{HEPES}$ and $2.5 \mathrm{mg} / \mathrm{mL}$ BSA at $37^{\circ} \mathrm{C}$ for 1 hour under agitation. The digested marrow was then passed through $16 \mathrm{Ga}, 20 \mathrm{Ga}$ and $25 \mathrm{Ga}$ needles twice before filtered using a strainer mesh (Sigma-Aldrich S3770). The digest was then centrifuged at $300 \mathrm{~g}$ for 10 minutes. The cell pellet was washed twice with DMEM supplemented with $10 \%$ FBS. Red blood cells were lysed using erythrocyte-lysing buffer $(0.154 \mathrm{M}$ ammonium chloride, $10 \mathrm{mM}$ potassium bicarbonate and $0.1 \mathrm{mM}$ EDTA) under gentle agitation for 10 minutes at room temperature. BMSCs were filtered through a $100 \mu \mathrm{m}$ nylon filter, washed in PBS, then resuspended in growth medium consisting of DMEM supplemented with $10 \% \mathrm{FBS}$. Isolated BMSCs were plated in $75 \mathrm{~cm}^{2}$ flasks and grown in DMEM supplemented with $10 \% \mathrm{FBS}, 1 \%$ antibiotics/antimitotics, and $1 \mathrm{ng} / \mathrm{mL}$ bFGF. After 4 days of culture, non-adherent cells were removed by changing the culture medium. The medium was then changed every 2-3 days and the cells were passaged twice before use. Cell cultures from each animal donor were maintained separately.

\section{Isolation of bovine adipose-derived stem cells}

Bovine ASCs were extracted under sterile conditions from the interdigital fat pads found in the adult hooves obtained from the same cows that the bone marrow was obtained. Cell isolation was performed as previously described [17]. Briefly, the fat pads were minced and digested under agitation at $37^{\circ} \mathrm{C}$ for 1 hour with $2 \%$ collagenase VIII in Krebs-Ringer buffer containing $3 \mathrm{mM}$ glucose, $25 \mathrm{mM}$ HEPES and $20 \mathrm{mg} / \mathrm{mL}$ BSA. The digested fat was filtered using a strainer mesh and pelleted by centrifugation at $1200 \mathrm{~g}$ for 5 minutes. Red blood cells were lysed using erythrocyte lysing buffer under gentle agitation for 10 minutes at room temperature. ASCs were filtered through a $100 \mu \mathrm{m}$ nylon filter, washed in PBS, then resuspended in growth medium consisting of DMEM:Ham's F-12 supplemented with $10 \% \mathrm{FBS}$ and $1 \%$ antibiotics/antimitotics. Isolated ASCs were plated in $75 \mathrm{~cm}^{2}$ flasks with the medium changed every 2-3 days and passaged up to 5 times. Cell cultures from each donor were maintained separately.

\section{Isolation of chondrocytes}

Primary articular chondrocytes were isolated from the metacarpal-phalangeal joint as described previously $[17,19]$. Briefly, the extracted cartilage was digested in protease $(0.5 \%$ protease in Ham's F-12 medium) at $37^{\circ} \mathrm{C}$ for $2 \mathrm{~h}$. Samples were then washed twice in Ham's F-12 medium and digested with $0.15 \%$ collagenase A in Ham's F-12 medium at $37^{\circ} \mathrm{C}$ for $18 \mathrm{~h}$. The digest was then filtered in a strainer mesh (Sigma-Aldrich S4020), washed in medium and centrifuged at $600 \mathrm{~g}$ for 6 minutes. Isolated chondrocytes were freshly used without passaging. 


\section{Characterization of BMSCs and ASCs}

To assess the differentiation potential of the BMSCs and ASCs, the isolated cells were grown in osteogenic, adipogenic and chondrogenic differentiation medium. For osteogenic differentiation, the cells were plated at 50,000 cells $/ \mathrm{cm}^{2}$ and cultured in DMEM supplemented with $10 \% \mathrm{FBS}, 50 \mu \mathrm{M}$ ascorbate-2-phosphate, $10 \mathrm{mM} \beta$-glycerophosphate and 100nM dexamethasone [20] for 21 days. Osteogenic differentiation potential was then assessed by Von Kossa staining. For adipogenic differentiation, the cells (plated at 50,000 cells $/ \mathrm{cm}^{2}$ ) were cultured in adipogenic induction medium (Gibco, A10070-01) for 14 days. Adipogenic differentiation potential was then assessed by Oil Red O staining. For chondrogenic differentiation, cell pellets $\left(1 \times 10^{6}\right.$ cells) were created by centrifugation ( $800 \mathrm{~g}$ for 3 minutes). The pellets were then transferred into 24 -well plates coated with $2 \%$ low melting agarose gel (Sigma-Aldrich, A6560). After 24 hours, the pellets were cultured in Ham's F-12 medium supplemented with $10 \mathrm{ng} / \mathrm{mL}$ TGF- $\beta 1,50 \mu \mathrm{g} / \mathrm{mL}$ ascorbate-2-phosphate, $6.25 \mu \mathrm{g} /$ $\mathrm{mL}$ bovine insulin, and 100nM dexamethasone [21] for 14 and 28 days. Chondrogenic differentiation potential was then assessed by DNA and sulphated GAG accumulation, as described below in the Biochemical Analyses section. For all cultures, the medium was changed 3 times per week.

\section{Direct and indirect co-cultures}

Two co-culture experiments were performed: direct and indirect co-culture. For direct co-culture, 3D cell pellets were created containing a total of $1 \times 10^{6}$ cells/pellet with different proportions of stem cells to mature chondrocytes: chondrocytes only (Chondrocytes), 25\% BMSCs and $75 \%$ chondrocytes (Direct BMSC 25/75), 50\% BMSCs and 50\% chondrocytes (Direct BMSC 50/50), 75\% BMSCs and 25\% chondrocytes (Direct BMSC 75/25), and 50\% ASCs and 50\% chondrocytes (Direct ASC $50 / 50$ ). Cell pellets were created by centrifugation ( $800 \mathrm{~g}$ for 3 minutes) and then transferred into 24-well culture plates coated with $2 \%$ low melting agarose. For indirect co-culture, individual 3D cell pellets $\left(1 \times 10^{6}\right.$ cells/pellet) were created with $100 \%$ stem cells and $100 \%$ chondrocytes and cultured together in 24-well culture plates, but separated by a transwell culture insert $(0.4 \mu \mathrm{m}$ pore size; PICM01250, Millicell. MA USA). Indirect groups include: pellet of BMSCs with pellet of chondrocytes (Indirect BMSC) and pellet of ASCs with pellet of chondrocytes (Indirect ASC). All co-cultures were grown in DMEM:Ham's F-12 medium supplemented with $10 \%$ FBS and $1 \%$ antibiotics/antimitotics for 14 or 28 days with the medium changed every 2-3 days.

\section{Histological and immunohistochemical analysis Oil red $O$ staining}

Intracellular lipid accumulation was assessed by Oil Red $\mathrm{O}$ staining after 14 days of adipogenic induction. Cultures were rinsed thoroughly with PBS and fixed for 10 minutes in 4\% paraformaldehyde in PBS. Oil Red O stock solution $(3 \mathrm{~g} / \mathrm{L}$ in isopropanol) was prepared as described previously [22]. Then, $2 \mathrm{~mL}$ Oil Red O working solution (stock solution diluted 3:2 in deionized water) was applied to each well for 5 minutes, followed by several washes with distilled water and counterstaining with hematoxylin for 3 minutes.

\section{Von kossa staining}

Calcium accumulation was assessed by Von Kossa staining after 21 days of osteogenic induction, as described previously $[17,23]$. Cultures were rinsed thoroughly with PBS and fixed for 10 minutes in $4 \%$ paraformaldehyde in PBS. Cultures were then rinsed with distilled water, stained with $1 \%$ silver nitrate in distilled water, incubated under UV light for 1 hour, incubated in 5\% sodium thiosulphate in distilled water for 5 minutes, and counterstained with hematoxylin for 3 minutes.

\section{Safranin-O staining}

Safranin-O staining was performed after 28 days of pellet culture, as described previously [24]. Cell pellets were fixed in $4 \%$ paraformaldehyde in PBS at $4^{\circ} \mathrm{C}$ overnight, dehydrated in graded ethanol solutions and embedded in paraffin at $65^{\circ} \mathrm{C}$. Sections, $5 \mu \mathrm{m}$ thick, were cut and mounted on Superfrost slides and dried at $37^{\circ} \mathrm{C}$ overnight. Sections were stained with safranin-O, which is a proteoglycan stain.

\section{Immunohistochemical staining for collagen I and II}

Immunohistochemical localization of collagen I and II was performed as previously described [24,25]. Briefly, cell pellets were fixed in $4 \%$ paraformaldehyde in PBS at $4^{\circ} \mathrm{C}$ overnight, dehydrated in graded ethanol solutions and embedded in paraffin at $65^{\circ} \mathrm{C}$. Sections were cut at $5 \mu \mathrm{m}$ thickness and mounted on Superfrost slides. The sections were deparaffinized and rehydrated, and then enzymatically treated with $0.05 \%$ trypsin ( $\mathrm{pH} \mathrm{7.8)}$ for 30 minutes at $37^{\circ} \mathrm{C}$ to facilitate antibody binding. They were also treated with $1 \%$ hydrogen peroxide and $1 \%$ BSA in PBS for 30 minutes to block endogenous peroxidase activity. Sections were then incubated with mouse

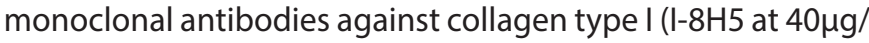
$\mathrm{mL}$; Daiichi Fine Chemicals Co Ltd, Japan) or collagen type II (II-II6B3 at $187 \mu \mathrm{g} / \mathrm{mL}$; Developmental Studies Hybridoma Bank, lowa, USA), diluted in PBS containing 1\% BSA, overnight at $4^{\circ} \mathrm{C}$. After the incubation with primary antibodies, sections were rinsed with PBS and incubated with biotinylated antimouse secondary antibodies using the Vectastain Elite ABC kit (Vector Laboratories Inc., Burlingame, USA) for 2 hours at room temperature. This was followed by incubation with diaminobenzidine for 6 minutes at room temperature. The sections were counterstained with Harris hematoxylin and mounted with permanent mounting medium. Stained sections were imaged using a Zeiss Axio-Image M1 microscope. Non-specific staining was assessed by omitting the primary antibody during staining. All experiments were performed at least three times with no detection of positive staining in the negative controls. 


\section{Biochemical analyses}

DNA, sulphated GAG and collagen contents of the cultures were quantified biochemically, as described previously [24]. Harvested cell pellets were washed in PBS, digested with papain $(40 \mu \mathrm{g} / \mathrm{mL}$ in $20 \mathrm{mM}$ ammonium acetate, $1 \mathrm{mM}$ EDTA and $2 \mathrm{mM}$ dithiothreitol) for 72 hours at $65^{\circ} \mathrm{C}$, and stored at $-20^{\circ} \mathrm{C}$ until analysis. Separate aliquots of the digest were assayed for DNA, GAG and collagen contents. The DNA content was quantified using Hoechst 33258 dye with calf thymus DNA as the standard [26]. The proteoglycan content was estimated by quantifying the amount of sulphated GAGs using 1,9-dimethylmethylene blue (DMMB) dye binding assay with chondroitin sulfate sodium salt from bovine cartilage as the standard $[\mathbf{2 7}, \mathbf{2 8}]$. Total collagen content was determined by quantifying the amount of hydroxyproline. Briefly, aliquots of the papain digested samples were hydrolyzed in $6 \mathrm{~N} \mathrm{HCl}$ for 18 hours at $110^{\circ} \mathrm{C}$, and the hydroxyproline content was determined in the hydrolyzed samples using chloramine-T/ Ehrlich's reagent assay [29]. The total amount of collagen was calculated assuming the hydroxyproline accounts for $10 \%$ of the total mass of collagen in cartilage [30].

\section{Statistical analyses}

Three separate batches of cells were used to create the cultures $(\mathrm{N}=3$ animal donors per isolation) and statistical analyses were conducted on the pooled results of the replicate samples (total of $n=5$ samples/group). Results were expressed as the mean \pm standard error of the mean (SEM). Biochemical quantification data was analyzed using one-way ANOVA with Tukey's post-hoc tests. Data was checked for both normality and equal variance prior to performing the statistical tests. Statistical tests were conducted using statistical software (GraphPad Prism 6) and significance was associated with $p$-values less than 0.05 .

\section{Results \\ Differentiation potential of BMSCs and ASCs}

To confirm their differentiation potential, both BMSCs and ASCs (at passage 2) were cultivated in osteogenic, adipogenic and chondrogenic differentiation media. After 21 days in osteogenic medium, there was an increase in calcium accumulation in the cytoplasm for both cell types as evaluated by von Kossa staining (Supplementary Figures 1A and 1B). While the induced BMSCs and ASCs both showed characteristic elongated fibroblast-like morphology, the differentiated BMSCs appeared to display more calcium deposition compared to the differentiated ASCs. After 14 days of cultivation in adipogenic medium, similar levels of intracellular lipid accumulation in BMSCs and ASCs were observed by Oil Red O staining (Supplementary Figures 1C and 1D). In these cultures, there appeared to be cells with two distinct phenotypes: (i) cells with unilocular morphology and large singular lipid droplets in the cytoplasm, and (ii) cells containing multiple, small lipid droplets located around the nucleus. Undifferentiated BMSCs and ASCs stained negatively for calcium and intracellular lipid accumulation (data not shown). For chondrogenic differentiation potential, BMSC and ASC cell pellets cultured for 28 days in chondrogenic medium were analyzed for DNA content and sulphated GAG accumulation (Supplementary Figures 1E and 1F). Differentiated BMSC and ASC pellets contained higher proportions of DNA and GAG, respectively, compared to their respective undifferentiated cultures $(p<0.05)$.

\section{Direct and indirect co-cultures of BMSCs and ASCs with chondrocytes}

Direct co-culture of BMSCs with chondrocytes (50:50) led to higher DNA, GAG and collagen contents compared to indirect co-culture (Figures 1A-1C; $p<0.05$ ). For the ASC co-cultures, while

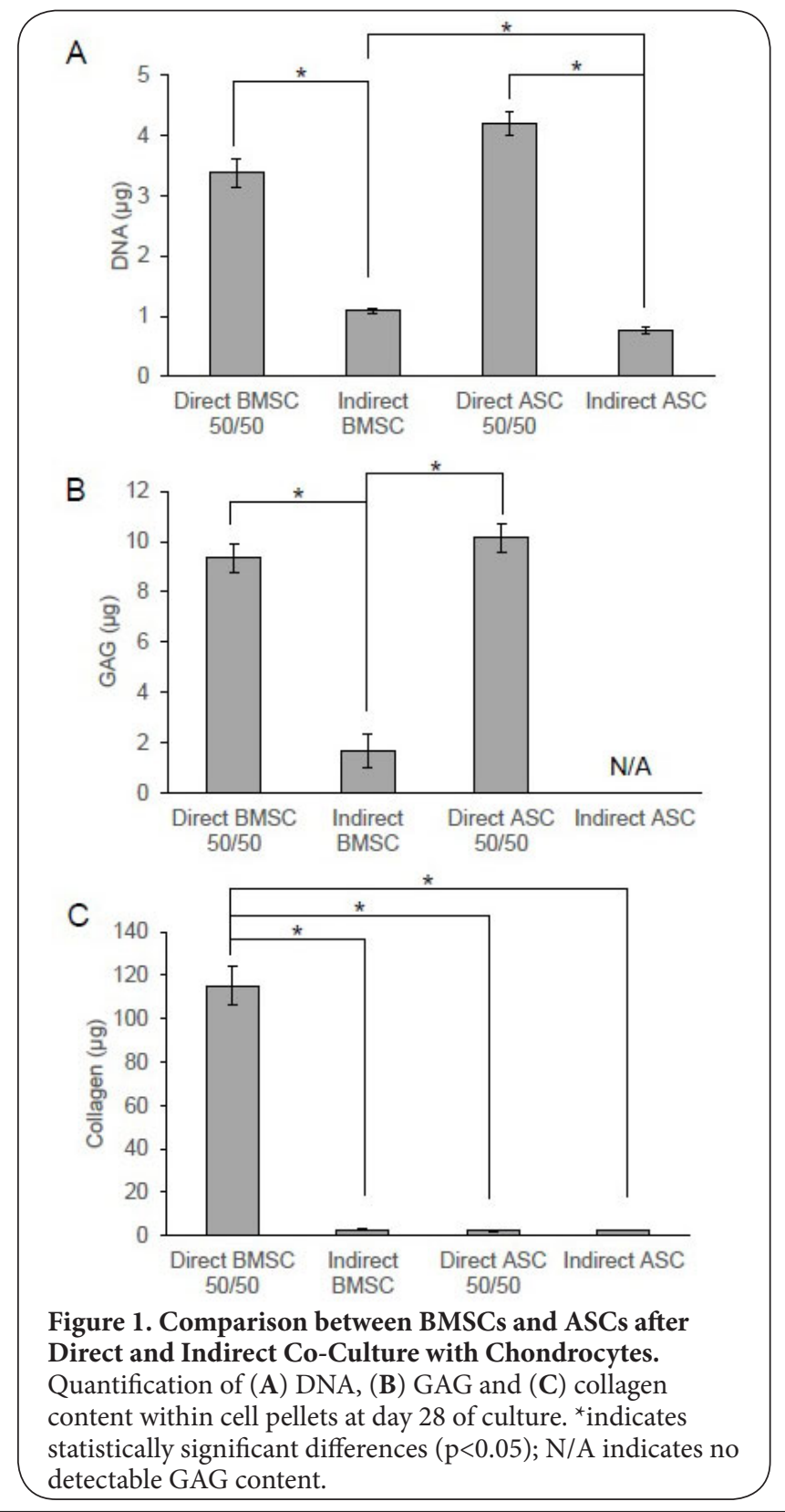


Chiu et al. Journal of Regenerative Medicine \& Tissue Engineering 2016, http://www.hoajonline.com/journals/pdf/2050-1218-5-1.pdf

the DNA and GAG contents were higher in direct co-culture (50:50) compared to indirect co-culture $(p<0.05)$, there was no significant difference in collagen content between direct and indirect co-cultures of ASCs $(p=0.22)$. Contrasting the results between the BMSC and ASC co-cultures, similar GAG contents were observed in both the BMSC and ASC direct co-cultures (Figure 1B). However, all ASC co-cultures (direct and indirect) displayed significantly lower collagen contents compared to the BMSC direct co-culture (Figure $1 \mathrm{C} ; p<0.05$ ). The collagento-GAG ratio of ASC direct co-culture was significantly lower than that of BMSC direct co-culture (Supplementary Figure 2; $\mathrm{p}<0.05)$. The DNA content in ASC indirect co-culture was also lower than BMSC indirect co-culture (Figure $1 \mathbf{A} ; p<0.05$ ).

\section{Effect of BMSCs and chondrocyte ratio in direct co- culture}

As the ASCs generally displayed poor chondrogenic potential, the influence of proportion of stem cells to mature chondrocytes in direct co-culture was examined further with BMSCs. In these experiments, BMSCs were directly co-cultured with chondrocytes in varying ratios of 1:3, 1:1 and 3:1 BMSCs to chondrocytes and compared to indirect co-culture of BMSCs and chondrocytes, BMSCs differentiated with chondrogenic medium, and chondrocyte-only cell pellets.

The DNA content of the differentiated BMSCs, chondrocyteonly, and all direct co-culture groups were similar and significantly higher than the undifferentiated BMSCs and indirect co-culture groups (Figure $2 \mathrm{~A} ; p<0.05$ ). The same trends were observed with the accumulation of GAG and collagen with similar chondrogenic ECM accumulation in the differentiated BMSCs, chondrocyte-only, and all direct co-culture groups, which was significantly higher than the undifferentiated and indirect co-culture groups (Figures $\mathbf{2 B}$ and $\mathbf{2 C} ; p<0.05$ ).

The presence of sulphated GAG accumulation was further supported by Safranin-O staining (Figure 3). Similar to the biochemical accumulation results, undifferentiated BMSC and indirect BMSC co-culture groups displayed no detectable staining, and positive staining was seen in the differentiated BMSCs, chondrocyte-only, and all direct co-culture groups (Figures 3A-3G). Interestingly, GAG staining in the direct co-culture groups appeared more prominent compared to the differentiated BMSCs. The proportion of BMSCs to chondrocytes also appeared to have an effect on GAG accumulation. Co-cultures with lower proportions of BMSCs (1:3 and 1:1) displayed GAG staining primarily concentrated near the periphery of the pellets (Figures $\mathbf{3} \mathbf{H}$ and $\mathbf{3 l}$ ), while more uniform GAG staining was observed in the cultures with a higher proportion of BMSCs (3:1) (Figure 3J).

Immunohistochemical staining for collagen I was evident in all cultures (undifferentiated BMSCs, differentiated BMSCs, and direct co-cultures) at day 14 of culture; however, collagen I expression was dramatically reduced in all cultures by day 28 with little-to-no staining evident (Figure 4). Similarly, collagen II expression was virtually non-existent at day 14 of

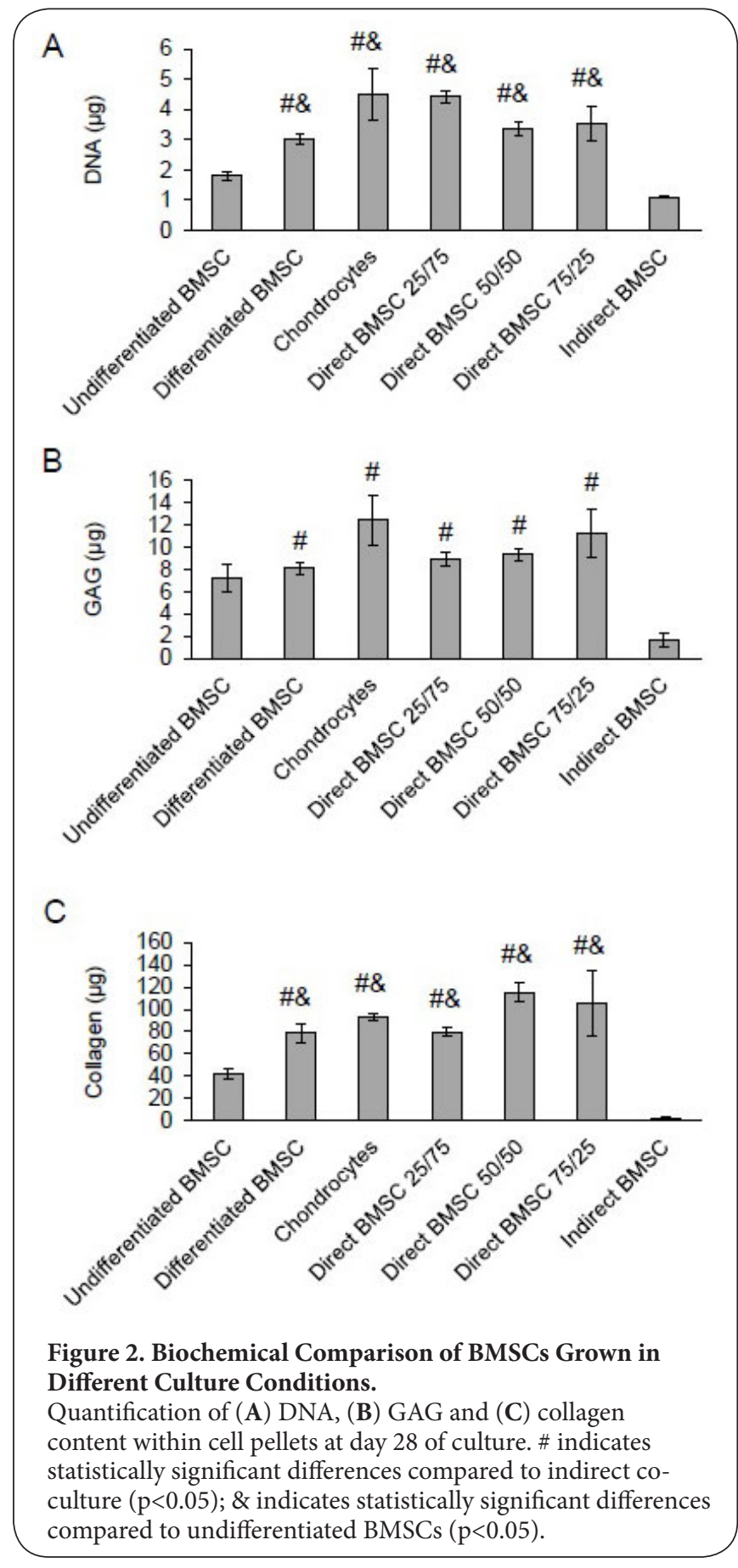

culture and the presence of positive collagen II staining was only found in the BMSC direct co-cultures by day 28 (Figure 5).

\section{Discussion}

The use of stem cells in cartilage tissue engineering, while advantageous for the proliferative and regenerative capacities, is hampered by the requirement of multiple growth factors to induce differentiation. Alternatively, differentiation in the 


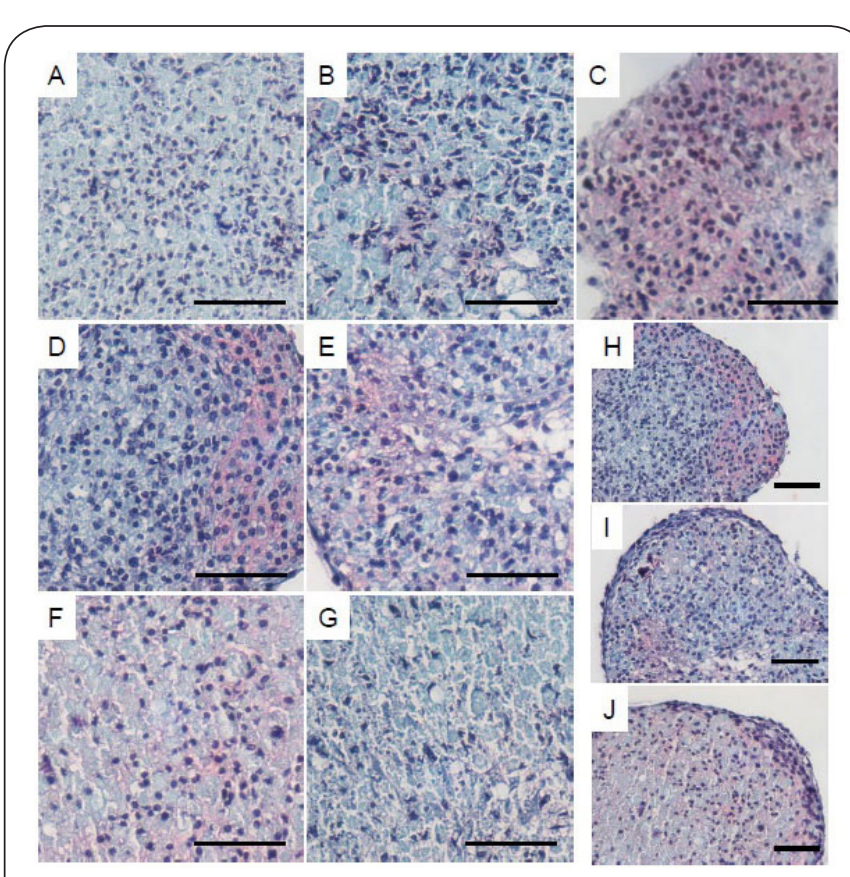

Figure 3. Safranin-O Staining of Sulphated Proteoglycans. Representative high magnification microscopy images of (A) Undifferentiated BMSC, (B) Differentiated BMSC, (C) Chondrocytes, (D) Direct BMSC 25/75, (E) Direct BMSC 50/50, (F) Direct BMSC 75/25, (G) Indirect BMSC at day 28 of culture. Lower magnification microscopy images of $(\mathbf{H})$ Direct BMSC 25/75, (I) Direct BMSC 50/50, and (J) Direct BMSC $75 / 25$ groups, showing localization of proteoglycans within pellets (periphery in Direct BMSC 25/75 and Direct BMSC 50/50 groups vs. uniform distribution in Direct BMSC 75/25 group). Scale bar $=100 \mu \mathrm{m}$.

absence of growth factors can be achieved by co-culturing stem cells with mature cells [11]. While promising results have been achieved for cartilage tissue engineering, it is not clear which co-culturing method or stem cell population(s) produce superior results. Thus, in this study, we compared direct and indirect co-cultures of BMSCs and ASCs with chondrocytes, and determined a suitable ratio of stem cells to chondrocytes in 3D scaffold-free culture.

During the investigation of co-culturing method, while there was no difference in the GAG or DNA contents of BMSC and ASC direct co-cultures, both direct co-cultures showed higher matrix contents than indirect co-cultures. This suggests that direct co-culture of stem cells with chondrocytes improves chondrogenic differentiation compared to indirect co-culture. The direct co-culture of BMSCs or ASCs with mature chondrocytes promotes their chondrogenic differentiation likely due to the secretion of growth factors such as TGF- $\beta$ and insulin-like growth factor-1 (IGF-1) by chondrocytes. Previously, Zuo et al., reported that direct 2D co-culture of rat BMSCs and articular chondrocytes yielded higher expressions of SOX-9, collagen II and aggrecan compared to indirect co-culture, showing that reciprocal interactions between chondrocytes

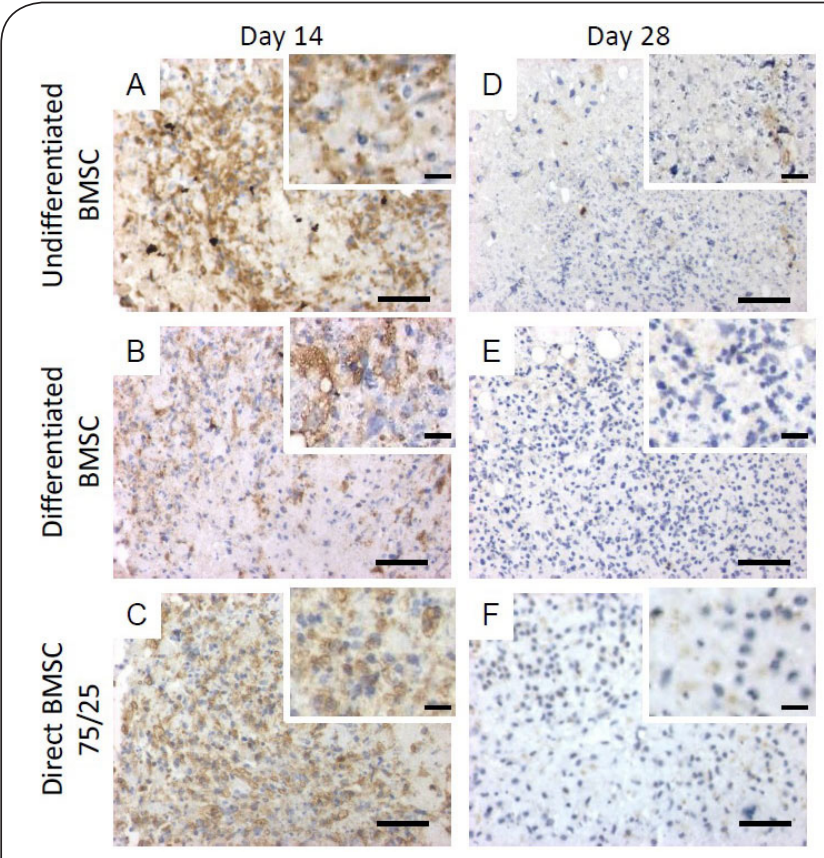

Figure 4. Collagen I Expression.

Representative microscopy images of Undifferentiated BMSC, Differentiated BMSC and Direct BMSC 75/25 groups at

(A-C) day 14 and (D-F) day 28. Collagen I appears brown and counterstaining of nuclei appears blue. Insets show close-up views of collagen I staining. Scale bar $=100 \mu \mathrm{m}$.

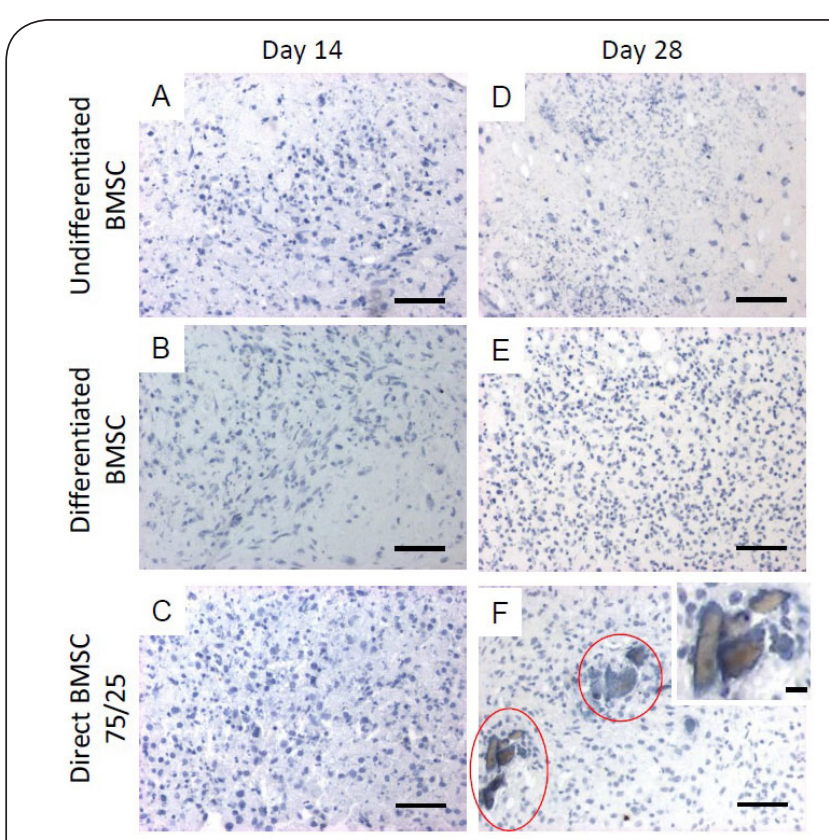

Figure 5. Collagen II Expression.

Representative microscopy images of Undifferentiated BMSC, Differentiated BMSC and Direct BMSC $75 / 25$ groups at (A-C) day 14 and (D-F) day 28. Red circles indicate positive staining (collagen II appears brown, counterstaining of nuclei appears blue). Inset shows a close-up view of positive collagen II staining in Direct BMSC 75/25 group at day 28. Scale bar $=100 \mu \mathrm{m}$. 
and BMSCs in co-culture involve both soluble factors and direct physical contact [14]. In vivo, the co-culture of BMSCs and chondrocytes in electrospun gelatin/polycaprolactone nanofibrous membranes subcutaneously in mice led to the formation of mature cartilage-like tissues similar to membranes seeded with chondrocytes only [13]. Lai et al., co-cultured ASCs with neonatal chondrocytes in 3D hydrogels using direct co-culture, indirect co-culture with the two cell types in separate layers and culture of each cell type alone with supplementation of conditioned medium from the other cell type [18]. Direct co-culture resulted in significantly enhanced chondrogenesis of ASCs and improved engineered cartilage tissues compared to indirect co-culture and cultures in conditioned medium. Additionally, increased ASC ratio in direct co-culture led to large neocartilage tissues due to increased synergy between ASCs and chondrocytes. In a separate study, the co-culture of human ASCs and porcine auricular chondrocytes in polyglycolic acid/polylactic acid scaffolds yielded cartilage-like tissues that contained abundant collagen II, similar to the positive control consisting of chondrocytes alone [16]. Although direct co-culturing of ASCs with chondrocytes showed some evidence of differentiation, these cultures accumulated significantly lower amounts of collagen compared to direct co-culture of BMSCs with chondrocytes. Collagen is an important extracellular matrix in cartilage tissues. Engineered cartilaginous tissues often have a low collagen-to-GAG ratio and in turn poor mechanical properties compared to the native cartilage [31]. The collagen content in tissue engineered cartilage constructs in previous studies was only $15-35 \%$ of the collagen content in the native cartilage [32]. This is potentially accounted for as GAGs tend to be first rapidly synthesized by the cells which may influence the collagen self-assembly process. Here, we found that ASCs is not an appropriate cell source to be differentiated in a co-culture with chondrocytes due to the resulting low collagen-to-GAG ratio. The collagen-to-GAG ratio of 1:5 in ASC direct co-culture was much lower than the 3:1 ratio in native bovine articular cartilage [33]. For this reason, these cells were not explored further.

The extent of differentiation in direct co-culturing of BMSCs with chondrocytes appeared to be comparable to that induced by growth factor stimulation. While positive staining of collagen I was evident in pellets of undifferentiated BMSCs cultures at day 14 , both growth factor differentiated BMSCs and direct co-culture of BMSCs with chondrocytes displayed lower detection of collagen I staining. Moreover, the expression of collagen I was reduced from day 14 to day 28 in these groups, thus indicating continuing differentiation with culture time. Interestingly however, only the direct co-culturing of BMSCs and chondrocytes displayed positive staining of collagen II at day 28. The presence of collagen II is a hallmark of chondrogenesis, with approximately $90 \%$ of the collagen in the cartilage extracellular matrix being collagen II [6]. The combination of matrix accumulation and the collagen types present in the cultures indicates that direct co-culture of undifferentiated BMSCs with chondrocytes can result in in vitro cultivated cartilage tissues similar to or better than those tissues grown with differentiated BMSCs or chondrocytes. Thus, this is a viable method to reduce the number of chondrocytes required in cartilage tissue engineering without the use of growth factors.

The next series of experiments investigated the effect of the proportions of BMSCs to chondrocytes in direct co-culture. While all BMSC direct co-cultures had similar DNA, GAG and collagen contents, higher proportions of BMSCs in the culture yielded a more uniform distribution of GAG. Various ratios of chondrocytes and BMSCs were previously co-cultured in $\mathrm{PCL}-$ PEG-PCL photocrosslinked hydrogels, and co-culture with a 1:4 chondrocyte-to-BMSC ratio was found to have increased GAG and collagen synthesis in vitro [12]. In vivo, this led to the formation of cartilage tissue with morphology similar to native hyaline cartilage. Kang et al., determined that a minimum of $30 \%$ chondrocytes was required to produce cartilage tissues with good shape retention, and engineered a human ear-shaped construct with 7:3 ratio of BMSCs to chondrocytes in polyglycolic acid/polylactic acid scaffolds [34]. In a 2D culture study, rabbit articular chondrocytes and BMSCs were co-cultured in ratios of 4:1, 2:1, 1:1, 1:2 and 1:4 [6]. The expression of collagen II and aggrecan was higher in 4:1, 2:1 and 1:1 groups compared to groups with chondrocytes or differentiated BMSCs only, with 2:1 being the best ratio of chondrocytes and BMSCs. Here, we found that the direct co-culture of BMSCs and chondrocytes at a ratio of 3:1 led to a uniform distribution of GAG while lower ratios of 1:3 and 1:1 showed a higher concentration of GAG in the periphery of the cell pellets. The different proportions of chondrocytes and BMSCs in the co-cultures may influence the interactions between the two cell types. It is likely that the pellets containing the higher proportions of chondrocytes led to Safranin-O staining concentrated near the periphery because the mature chondrocytes initially rapidly produced cartilaginous matrix rather than interacting with the BMSCs. This in turn reduced nutrient diffusion and hindered the differentiation of cells in the centre of the pellet. In contrast, the pellets containing the higher proportion of BMSCs had uniform Safranin-O staining because the BMSCs and chondrocytes were given a longer period of time to interact initially, and in turn the cells differentiated and distributed uniformly without diffusional limitations caused by matrix production.

Previous studies determining the optimal ratio of BMSCs-tochondrocytes for co-culture were performed as either $2 \mathrm{D}$ cultures or cultures in biomaterials. The present study was performed using a 3D scaffold-free approach, in which either stem cells alone or a combination of stem cells and chondrocytes were cultivated as cell pellets. It appears that our best condition (a ratio of 3:1) is in a similar range as studies done in biomaterials rather than those done as 2D cultures. Similarly, other studies utilizing 3D culturing methods have concluded that a higher 
proportion of BMSCs is required for optimal differentiation. This is in contrast with 2D culturing studies that indicate a higher number of chondrocytes (than BMSCs) leads to better cartilaginous extracellular matrix production [6]. While this suggests the need to optimize the ratio of stem cells to chondrocytes for specific co-culturing methods, it should be mentioned that our 3D scaffold-free culturing approach can be more easily translated into clinical applications and better mimics the native environment of the cells in vivo.

\section{Conclusions}

In this study, we investigated the chondrogenic differentiation potential of ASCs and BMSCs in direct and indirect coculture with mature chondrocytes. ASC co-cultures (direct and indirect) were found to be inferior to BMSC co-cultures and generally displayed significantly lower accumulation of collagen; suggesting that ASCs are not suitable for chondrogenic differentiation through co-culture with chondrocytes. While direct co-culture of BMSCs and chondrocytes was superior to indirect co-culture, there was also an observed influence of the proportion of stem cells to chondrocytes with higher proportions of BMSCs yielding a more uniform distribution of chondrogenic ECM. Thus, direct co-culture of BMSCs and chondrocytes appears to be a feasible approach to generate engineered cartilage tissues with reduced numbers of chondrocytes and without the need for growth factor differentiation. Future work will focus on determining the optimal ratio of human chondrocytes and BMSCs for chondrogenic differentiation under direct co-culture conditions, as well as elucidating the mechanisms mediating chondrogenic differentiation in this system.

\section{Additional files}

\section{Supplementary Figure 1}

Supplementary Figure 2

\section{Competing interests}

The authors declare that they have no competing interests.

Authors' contributions

\begin{tabular}{|l|c|c|c|c|c|}
\hline Authors' contributions & LLC & JB & RG & KC & SDW \\
\hline Research concept and design & -- & $\checkmark$ & $\checkmark$ & -- & $\checkmark$ \\
\hline Collection and/or assembly of data & $\checkmark$ & $\checkmark$ & $\checkmark$ & $\checkmark$ & -- \\
\hline Data analysis and interpretation & $\checkmark$ & $\checkmark$ & $\checkmark$ & -- & -- \\
\hline Writing the article & $\checkmark$ & -- & -- & -- & -- \\
\hline Critical revision of the article & $\checkmark$ & -- & -- & -- & $\checkmark$ \\
\hline Final approval of article & -- & -- & -- & -- & $\checkmark$ \\
\hline Statistical analysis & $\checkmark$ & -- & -- & -- & -- \\
\hline
\end{tabular}

\section{Acknowledgement}

This work was funded by the Canadian Institutes of Health Research.

\section{Publication history}

Editor: Jin Nam, University of California-Riverside, USA.

Michael Hadjiargyrou, New York Institute of Technology, USA.

Received: 17-Apr-2016 Final Revised: 13-May-2016

Accepted: 25-May-2016 Published: 07-Jun-2016

\section{References}

1. El Sayed K, Zreiqat H, Ertel W and Schulz-Tanzil G. Stimulated Chondrogenesis via Chondrocytes Co-culturing. Biochips \& Tissue chips. 2012; S2:003.

2. Xu T, Binder KW, Albanna MZ, Dice D, Zhao W, Yoo JJ and Atala A Hybrid printing of mechanically and biologically improved constructs for cartilage tissue engineering applications. Biofabrication. 2013; 5:015001. | Article | PubMed

3. Spiller KL, Liu Y, Holloway JL, Maher SA, Cao Y, Liu W, Zhou G and Lowman AM. A novel method for the direct fabrication of growth factor-loaded microspheres within porous nondegradable hydrogels: controlled release for cartilage tissue engineering. J Control Release. 2012; 157:3945. | Article | PubMed

4. Chahine NO, Collette NM, Thomas CB, Genetos DC and Loots GG. Nanocomposite scaffold for chondrocyte growth and cartilage tissue engineering: effects of carbon nanotube surface functionalization. Tissue Eng Part A. 2014; 20:2305-15. | Article | PubMed Abstract | PubMed FullText

5. Schwarz S, Koerber L, Elsaesser AF, Goldberg-Bockhorn E, Seitz AM, Durselen L, Ignatius A, Walther P, Breiter R and Rotter N. Decellularized cartilage matrix as a novel biomatrix for cartilage tissue-engineering applications. Tissue Eng Part A. 2012; 18:2195-209. I Article I PubMed

6. Qing C, Wei-ding C and Wei-min F. Co-culture of chondrocytes and bone marrow mesenchymal stem cells in vitro enhances the expression of cartilaginous extracellular matrix components. Braz J Med Biol Res. 2011; 44:303-10. I PubMed

7. Solchaga LA, Penick KJ and Welter JF. Chondrogenic differentiation of bone marrow-derived mesenchymal stem cells: tips and tricks. Methods Mol Biol. 2011; 698:253-78. | Article | PubMed Abstract | PubMed FullText

8. Murdoch AD, Grady LM, Ablett MP, Katopodi T, Meadows RS and Hardingham TE. Chondrogenic differentiation of human bone marrow stem cells in transwell cultures: generation of scaffold-free cartilage. Stem Cells. 2007; 25:2786-96. | Article | PubMed

9. Barry F, Boynton RE, Liu B and Murphy JM. Chondrogenic differentiation of mesenchymal stem cells from bone marrow: differentiationdependent gene expression of matrix components. Exp Cell Res. 2001: 268:189-200. | Article | PubMed

10. Estes BT, Diekman BO, Gimble JM and Guilak F. Isolation of adiposederived stem cells and their induction to a chondrogenic phenotype. Nat Protoc. 2010; 5:1294-311. | Article | PubMed Abstract | PubMed FullText

11. Paschos NK, Brown WE, Eswaramoorthy R, Hu JC and Athanasiou KA. Advances in tissue engineering through stem cell-based co-culture. $J$ Tissue Eng Regen Med. 2015; 9:488-503. | Article I PubMed

12. Ko CY, Ku KL, Yang SR, Lin TY, Peng S, Peng YS, Cheng MH and Chu IM. In vitro and in vivo co-culture of chondrocytes and bone marrow stem cells in photocrosslinked PCL-PEG-PCL hydrogels enhances cartilage formation. J Tissue Eng Regen Med. 2013. I Article I PubMed

13. He X, Feng B, Huang C, Wang H, Ge Y, Hu R, Yin M, Xu Z, Wang W, Fu $\mathrm{W}$ and Zheng J. Electrospun gelatin/polycaprolactone nanofibrous membranes combined with a coculture of bone marrow stromal cells and chondrocytes for cartilage engineering. Int J Nanomedicine. 2015; 10:2089-99. | Article | PubMed Abstract | PubMed FullText

14. Zuo Q, Cui W, Liu F, Wang Q, Chen Z and Fan W. Co-cultivated mesenchymal stem cells support chondrocytic differentiation of articular chondrocytes. Int Orthop. 2013; 37:747-52. I Article I PubMed Abstract | PubMed FullText

15. Levorson EJ, Santoro M, Kasper FK and Mikos AG. Direct and indirect coculture of chondrocytes and mesenchymal stem cells for the generation of polymer/extracellular matrix hybrid constructs. Acta Biomater. 2014; 10:1824-35. | Article | PubMed Abstract | PubMed FullText

16. Lv X, Zhou G, Liu X, Liu H, Chen J, Liu K and Cao Y. Chondrogenesis by co-culture of adipose-derived stromal cells and chondrocytes in vitro. Connect Tissue Res. 2012; 53:492-7. I Article I PubMed

17. Zhao Y, Waldman SD and Flynn LE. Multilineage co-culture of adiposederived stem cells for tissue engineering. J Tissue Eng Regen Med. 2015; 9:826-37. | Article | PubMed

18. Lai JH, Kajiyama G, Smith RL, Maloney W and Yang F. Stem cells 
Chiu et al. Journal of Regenerative Medicine \& Tissue Engineering 2016, http://www.hoajonline.com/journals/pdf/2050-1218-5-1.pdf

catalyze cartilage formation by neonatal articular chondrocytes in 3D biomimetic hydrogels. Sci Rep. 2013; 3:3553. | Article | PubMed

19. Boyle J, Luan B, Cruz TF and Kandel RA. Characterization of proteoglycan accumulation during formation of cartilagenous tissue in vitro. Osteoarthritis Cartilage. 1995; 3:117-25. | Article | PubMed

20. Zuk PA, Zhu M, Mizuno H, Huang J, Futrell JW, Katz AJ, Benhaim P, Lorenz HP and Hedrick MH. Multilineage cells from human adipose tissue: implications for cell-based therapies. Tissue Eng. 2001; 7:211-28. | Article | PubMed

21. Neupane M, Chang CC, Kiupel M and Yuzbasiyan-Gurkan V. Isolation and characterization of canine adipose-derived mesenchymal stem cells. Tissue Eng Part A. 2008; 14:1007-15. | Article | PubMed

22. Flynn L, Prestwich GD, Semple JL and Woodhouse KA. Adipose tissue engineering with naturally derived scaffolds and adipose-derived stem cells. Biomaterials. 2007; 28:3834-42. | Article | PubMed

23. Zhao Y, Waldman SD and Flynn LE. The effect of serial passaging on the proliferation and differentiation of bovine adipose-derived stem cells. Cells Tissues Organs. 2012; 195:414-27. | Article | PubMed

24. Rosa RG, Joazeiro PP, Bianco J, Kunz M, Weber JF and Waldman SD. Growth factor stimulation improves the structure and properties of scaffold-free engineered auricular cartilage constructs. PLOS One. 2014; 9:e105170. | Article | PubMed Abstract | PubMed FullText

25. Rosa RG, Collavino K, Lakhani A, Delve E, Weber JF, Rosenthal AK and Waldman SD. Clodronate exerts an anabolic effect on articular chondrocytes mediated through the purinergic receptor pathway. Osteoarthritis Cartilage. 2014; 22:1327-36. | Article | PubMed

26. Kim YJ, Sah RL, Doong JY and Grodzinsky AJ. Fluorometric assay of DNA in cartilage explants using Hoechst 33258. Anal Biochem. 1988; 174:16876. | Article | PubMed

27. Farndale RW, Buttle DJ and Barrett AJ. Improved quantitation and discrimination of sulphated glycosaminoglycans by use of dimethylmethylene blue. Biochim Biophys Acta. 1986; 883:173-7. | Article | PubMed

28. Goldberg RL and Kolibas LM. An improved method for determining proteoglycans synthesized by chondrocytes in culture. Connect Tissue Res. 1990; 24:265-75. | Article | PubMed

29. Woessner JF, Jr. The determination of hydroxyproline in tissue and protein samples containing small proportions of this imino acid. Arch Biochem Biophys. 1961; 93:440-7. | Article | PubMed

30. Heinegard D, Bayliss M and Lorenzo P. Biochemistry and Metabolism of Normal and Osteoarthritic Cartilage. Osteoarthritis. 1998; 74-84.

31. Elder $S$ and Thomason J. Effect of platelet-rich plasma on chondrogenic differentiation in three-dimensional culture. Open Orthop J. 2014; 8:7884. | Article | PubMed Abstract | PubMed FullText

32. Kock $L$, van Donkelaar $C C$ and Ito $K$. Tissue engineering of functional articular cartilage: the current status. Cell Tissue Res. 2012; 347:613-27. | Article | PubMed Abstract | PubMed FullText

33. Waldman SD, Grynpas MD, Pilliar RM and Kandel RA. Characterization of cartilagenous tissue formed on calcium polyphosphate substrates in vitro. J Biomed Mater Res. 2002; 62:323-30. | Article | PubMed

34. Kang N, Liu X, Yan L, Wang Q, Cao Y and Xiao R. Different ratios of bone marrow mesenchymal stem cells and chondrocytes used in tissue-engineered cartilage and its application for human ear-shaped substitutes in vitro. Cells Tissues Organs. 2013; 198:357-66. | Article | PubMed

\section{Citation:}

Chiu LLY, Bianco J, Giardini-Rosa R, Collavino K and Waldman SD. Direct and indirect co-culture of bone marrow stem cells and adipose-derived stem cells with chondrocytes in 3D scaffold-free culture. J Regen Med Tissue Eng. 2016; 5:1.

http://dx.doi.org/10.7243/2050-1218-5-1 